Pacific Journal of Mathematics

ON UNIMODULAR MATRICES 


\section{ON UNIMODULAR MATRICES}

\section{Heller and A. J. Hoffman}

1. Introduction and summary. For the purpose of this note a matrix is called unimodular if every minor determinant equals 0,1 or -1 .

I. Heller and C. B. Tompkins [1] have considered a set

$$
S=\left\{u_{i}, v_{j}, u_{i}+v_{j}, u_{i}-u_{i *}, v_{j}-v_{j *}\right\}
$$

where the $u_{1}, u_{2}, \cdots, u_{m}, v_{1}, v_{2}, \cdots, v_{n}$ are linearly independent vectors in $m+n=k$-dimensional space $E$, and have shown that in the coordinate representation of $S$ with respect to an arbitrary basis in $E$ every nonvanishing determinant of $k$ vectors of $S$ has the same absolute value, and that, with respect to a basis in $S$, the vectors of $S$ or of any subset of $S$ are the columns of a unimodular matrix. For the purpose of this note the class of unimodular matrices obtained in this fashion shall be denoted as the class $T$.

A. J. Hoffman and J. B. Kruskal [4] have considered incidence matrices $A$ of vertices versus directed paths of an oriented graph $G$, and proved that:

(i) if $G$ is alternating, then $A$ is unimodular;

(ii) if the matrix $A$ of all directed paths of $G$ is unimodular, then $G$ is alternating. The terms are defined as follows. A graph $G$ is oriented if it has no circular edges, at most one edge between any given two vertices, and each edge is oriented. A path is a sequence of distinct vertices $v_{1}, v_{2}, \cdots, v_{k}$ of $G$ such that, for each $i$ from 1 to $k-1, G$ contains an edge connecting $v_{i}$ with $v_{i+1}$; if the orientation of these edges is from $v_{i}$ to $v_{i+1}$, the path is directed; if the orientation alternates throughout the sequence, the path is alternating. A loop is a sequence of vertices $v_{1}, v_{2}, \cdots, v_{k}$, which is a path except that $v_{k}=v_{1}$. A loop is alternating if successive edges are oppositely oriented and the first and last edges are oppositely oriented. The graph is alternating if every loop is alternating. The incidence matrix $A=\left(a_{i j}\right)$ of the vertices $v_{i}$ of $G$ versus a set of directed paths $p_{1}, p_{2}, \cdots, p_{k}$ of $G$ is defined by

$$
a_{i j}= \begin{cases}1 & \text { if } v_{i} \text { is in } p_{j} \\ 0 & \text { otherwise } .\end{cases}
$$

The class of unimodular matrices thus associated with alternating graphs shall be denoted by $K$.

I. Heller [2] and [3] has considered unimodular matrices obtained

Received April 12, 1962. Research sponsored (for both authors) by the RAND Corporation, in conjunction with the Symposium in Combinatorial Analysis, and (for the first author) by the National Science Foundation. 
by representing the edges (interpreted as vectors) of an $n$-simplex in terms of a basis chosen among the edges (in graph theoretical terms: the edges and vertices of the simplex form a complete graph $G$; a basis is a maximal tree in $G$, that is, a tree containing all vertices of $G$ ), and has shown that:

(i) the matrix representing all edges of the simplex is unimodular and maximal (i.e., will not remain unimodular when a new column is adjoined);

(ii) the columns of every unimodular matrix of $n$ rows and $n(n+1)$ columns represent the edges of an $n$-simplex.

The class of (unimodular) matrices whose columns are among the edges of a simplex shall be denoted by $H . \quad H$ can also be defined as a class of incidence matrices: A matrix $A$ belongs to $H$ if there is some oriented graph $F$ without loops such that $A$ is the incidence matrix of the edges of $F$ versus a set of path in $F$. That is,

$$
a_{i j}=\left\{\begin{aligned}
1 & \text { if edge } e_{i} \text { is in path } p_{j} \\
-1 & \text { if }-e_{i} \text { is in } p_{j} \\
0 & \text { otherwise }
\end{aligned}\right.
$$

In [2] it has further been shown that:

(iii) there exist unimodular matrices which do not belong to $H$;

(iv) the classes $H$ and $T$ are identical.

The purpose of the present note is to show that the class $K$ is identical with the set of nonnegative matrices of $H$.

2. THEOREM. If a matrix $A$ of $n$ rows and $m$ columns belongs to $K$ (i.e., $A$ is the incidence matrix of the $n$ vertices of some alternating graph $G$ versus a set of $m$ directed paths in $G$ ), then $A$ belongs to $H$ (i.e, there is some $n$-simplex $S$ and $a$ basis $B$ among its edges such that the columns of $A$ represent edges of $S$ in terms of $B$ ). Conversely, every non-negative matrix of $H$ belongs to $K$.

3. Notation. An oriented graph is viewed as a set

$$
R=V \cup E,
$$

where $V$ is the set of vertices $A_{1}, A_{2}, \cdots, A_{n}$, and $E$ is the set of oriented edges $e_{\nu}$, that is certain ordered pairs $\left(A_{i}, A_{j}\right)$ with $j \neq i$ of elements of $V$, such that at most one of the two pairs $\left(A_{i}, A_{j}\right),\left(A_{j}, A_{i}\right)$ is in $E$. For brevity of notation we define

$$
\left[A_{i}, A_{j}\right]=\left\{\left(A_{i}, A_{j}\right),\left(A_{j}, A_{i}\right)\right\} \text {. }
$$

The origin and endpoint of an edge $e$ are denoted by $\rho e$ and $\sigma e$ :

$$
\rho(A, B)=A, \quad \sigma(A, B)=B,
$$


If $A$ and $B$ are vertices of $R$, the relation $A \prec B$ ( $A$ is immediate predecessor of $B$ ), also written as $B>A$, is defined by

$$
A \prec B \Longleftrightarrow(A, B) \in R \text {. }
$$

Similarly, if $a, b$ are edges of $R$,

$$
a \prec b \Longleftrightarrow \sigma a=\rho b .
$$

A subset $V^{\prime}$ of vertices of $R$ defines a subgraph of $R$

$$
R\left(V^{\prime}\right)=V^{\prime} \cup E^{\prime}
$$

where $(A, B) \in E^{\prime} \Longleftrightarrow A \in V^{\prime}, B \in V^{\prime},(A, B) \in E$.

4. Proof. Using the graph-theoretical definition of the class $H$, the first half of the theorem shall be proved by showing that to each alternating graph $G$ there is an oriented loopless graph $F$ such that the $K$-matrices associated with $G$ are among the $H$-matrices associated with $F$.

A column of a $K$-matrix is the incidence column $K_{p}$ of the vertices of $G$ versus a directed path $p$ in $G$; a column of an $H$-matrix is the incidence column $H_{q}$ of the edges of $F$ versus a path $q$ in $F$. For given $G$ it will therefore be sufficient to show the existence of an $F$ such that

to each directed path $p$ in $G$ there is a path $q=\varphi(p)$ in $F$ such that $K_{p}=H_{q}$.

This will be shown by constructing an $F$ and a mapping $\varphi$ of the set of vertices of $G$ onto the set of edges of $F$ in such a way that $\varphi$ satisfies (4.1), or equivalently, that $\varphi$ preserves the relation defined in (3.4) and (3.5), that is, for any two distinct vertices $A, B$ of $G$,

$$
A \prec B \text { (in } G) \Longrightarrow \varphi(A) \prec \varphi(B) \text { (in } F \text { ) . }
$$

The construction of $F$ and $\varphi$ shall now be carried out under the assumption that $G$ is connected. If $G$ is not connected, the same construction can be applied to each component of $G$, yielding an $F$ with an equal number of components.

If $G$ has $n$ vertices, take as the vertices of $F$ a set of $n+1$ distinct elements $P_{0}, P_{1}, \cdots, P_{n}$.

The $n$ edges $e_{1}, e_{2}, \cdots, e_{n}$ of $F$ are defined successively as follows.

First, choose an arbitrary vertex $A_{1}$ in $G$, define

$$
\varphi\left(A_{1}\right)=e_{1}=\left(P_{0}, P_{1}\right),
$$

and note that:

(i) the subgraph $G_{1}=G\left(A_{1}\right)$, consisting of the one vertex $A_{1}$ of $G$, is, trivially, connected; 
(ii) the graph $F_{1}=\left\{P_{0}, P_{1},\left(P_{0}, P_{1}\right)\right\}$ is connected;

(iii) with respect to $G_{1}$ and $F_{1}, \varphi$ trivially satisfies (4.2).

Then, assuming $A_{\nu} \in G$ already chosen and $e_{\nu}=\varphi\left(A_{\nu}\right)$ defined for $\nu=1,2, \cdots, k$ in such a manner that $G_{k}=G\left\{A_{1}, A_{2}, \cdots, A_{k}\right\}$ and $F_{k}=$ $\left\{P_{0}, P_{1}, \cdots, P_{k}, e_{1}, \cdots, e_{k}\right\}$ are each connected and $\varphi$ satisfies (4.2) with respect to $G_{k}$ and $F_{k}$, choose $A_{k+1} \in G$ such that

$$
\left[A_{i}, A_{k+1}\right] \cap G \neq 0
$$

for some $i \leqq k$ and define

$$
\varphi\left(A_{k+1}\right)=e_{k+1}=\left\{\begin{array}{l}
\left(\sigma e_{i}, P_{k+1}\right) \text { when }\left(A_{i}, A_{k+1}\right) \in G \\
\left(P_{k+1}, \rho e_{i}\right) \text { when }\left(A_{k+1}, A_{i}\right) \in G,
\end{array}\right.
$$

noting that this definition depends on the choice of $i$ since more than one $i$ may satisfy (4.4).

Obviously, $G_{k+1}$ and $F_{k+1}$ are each connected.

To show that $\varphi$ satisfies (4.2) with respect to $G_{k+1}$ and $F_{k+1}$, let $A_{r} \prec A_{s}$ in $G_{k+1}$.

If $r \leqq k$ and $s \leqq h,(4.2)$ is satisfied according to the induction's hypothesis.

For $\{r, s\}=\{i, k+1\},(4.2)$ is satisfied by definition (4.5). Namely: for $r=i, s=k+1$, (4.5) defines $e_{k+1}=\left(\sigma e_{i}, P_{k+1}\right)$, hence $\sigma e_{i}=\rho e_{k+1}$, which by (3.5) means $e_{i} \prec e_{k+1}$; similarly for $s=i, r=k+1$, (4.5) defines $e_{k+1}=\left(P_{k+1}, \rho e_{i}\right)$, hence $\sigma e_{k+1}=\rho e_{i}$, which means $e_{k+1} \prec e_{i}$.

There remains the case $\{r, s\}=\{j, k+1\}, j \neq i, j \leqq k$, with

$$
\left[A_{j}, A_{k+1}\right] \cap G_{k+1} \neq 0 \text {, }
$$

that is either $A_{j} \prec A_{k+1}$ or $A_{k+1} \prec A_{j}$ in $G_{k+1}$.

In this case $A_{k+1}$, which by (4.4) has an edge in common with $A_{i}$, now also has an edge in common with $A_{j} \neq A_{i}$, thus connecting these two distinct vertices of $G_{k}$ by the path

$$
A_{i}, A_{k+1}, A_{j}
$$

in $G_{k+1}$ but outside $G_{k}$.

On the other hand, by the induction's hypothesis, $G_{k}$ is connected. Hence $A_{i}$ and $A_{j}$ are connected by a path in $G_{k}$

$$
A_{i}, A_{t_{1}}, A_{t_{2}}, \cdots, A_{t_{\lambda}}, A_{j}
$$

$(\lambda=0$ not a priori excluded $)$.

The paths (4.7) and (4.8) combine to the loop

$$
A_{k+1}, A_{i}, A_{t_{1}}, A_{t_{2}}, \cdots, A_{t_{\lambda}}, A_{j}, A_{k+1}
$$

in $G_{k+1}$, which is obviously also a loop in $G$. 
Since $G$ is alternating, the loop (4.9) must be alternating. This implies that the number of vertices is even, hence $\lambda=2 \nu+1$, and that the orientation is either

$$
A_{k+1} \prec A_{i}>A_{t_{1}} \prec A_{t_{2}} \succ \cdots \prec A_{t_{2 \nu}}>A_{t_{2 \nu+1}} \prec A_{j}>A_{k+1}
$$

or the opposite.

Now assume first

$$
A_{k+1} \prec A_{j},
$$

which implies the orientation (4.10), and consider that part of the loop which is in $G_{k}$, namely the path (4.8)

(4.10) and the induction's hypothesis that, relative to $G_{k}$ and $F_{k}, \varphi$ satisfies (4.2), imply

$$
e_{i}>e_{t_{1}} \prec e_{t_{2}}>\cdots \prec e_{t_{2 \nu}}>e_{t_{2 \nu+1}} \prec e_{j},
$$

hence

$$
\rho e_{i}=\sigma e_{t_{1}}=\rho e_{t_{2}}=\sigma e_{t_{3}}=\cdots=\rho e_{t_{2 \nu}}=\sigma e_{t_{2 \nu+1}}=\rho e_{j} .
$$

The definition (4.5) of $e_{k+1}$, in conjunction with $A_{k+1} \prec A_{i}$ from (4.10), implies

$$
\sigma e_{k+1}=\rho e_{i} .
$$

This together with (4.13) yields

$$
\sigma e_{k+1}=\rho e_{j}, \text { that is } e_{k+1} \prec e_{j},
$$

which proves that assumption (4.11) implies (4.15).

Similarly, the assumption $A_{k+1}>A_{j}$ yields $e_{k+1}>e_{j}$, by reversing the relation $\prec$ and interchanging $\rho$ and $\sigma$ in the above argument.

This completes the proof that to any connected alternating graph $G$ there exists a connected oriented graph $F$ and a mapping $\varphi$ satisfying (4.2)

That $F$ has no loops (and hence is a tree) is obvious from the fact that its $n+1$ vertices are connected by $n$ edges. Hence, the incidence matrices of $F$ certainly belong to class $H$.

If $G$ consists of $k$ components, the construction will yield an $F$ consisting of $k$ trees.

This completes the proof of the theorem's first half, namely that every $K$-matrix is an $H$-matrix.

The second half of the theorem, namely that each nonnegative $H$-matrix is a $K$-matrix, is due to J. Edmonds. It will be proved by showing that to each loopless oriented $F$ there is an alternating $G$ and a mapping $\psi$ of the edges of $F$ onto the vertices of $G$ that preserves the relation $\prec$, that is, for any two edges $a, b$ of $F$ 


$$
a \prec b \Longrightarrow \psi(a) \prec \psi(b) .
$$

This is achieved by the following simple construction.

If $F$ has $n$ edges $e_{1}, e_{2} \cdots, e_{n}$, choose a set of $n$ elements $A_{1}, A_{2}, \cdots, A_{n}$ as the vertices of $G$, define $\psi$ by

$$
\psi e_{i}=A_{i},
$$

and define the edges of $G$ by

$$
\left(A_{i}, A_{j}\right) \in G \Longleftrightarrow e_{i} \prec e_{j},
$$

that is, $G$ shall have an edge oriented from $A_{i}$ to $A_{j}$ if and only if $\sigma e_{i}=\rho e_{j}$.

Obviously $\psi$ preserves the relation $\prec$, since (4.18) is equivalent to

$$
A_{i} \prec A_{j} \Longleftrightarrow e_{i} \prec e_{j} .
$$

Note that $\prec$ is also preserved by the inverse of $\psi$, that is, in the transition from $G$ to $F$.

Note further that $G$ is oriented (in the sense of the definition given in [4] and cited in $\S 1$ of present note), that is:

(a) each edge of $G$ is oriented, since the edges of $G$ have been defined by (4.18) as oriented edges;

(b) $G$ has no circular edge, since $\left(A_{i}, A_{i}\right) \in G$ for some $i$ would imply $e_{i} \prec e_{i}$, or equivalently $\sigma e_{i}=\rho e_{i}$, that is, $e_{i}$ a circular edge in $F$, contradicting the assumption on $F$;

(c) $G$ has at most one edge between any given two vertices: $\left(A_{i}, A_{j}\right) \in G$ and $\left(A_{j}, A_{i}\right) \in G$ for some pair $i, j$, would imply $e_{i} \prec e_{j}$ and $e_{j} \prec e_{i}$, that is $\sigma e_{i}=\rho e_{j}$ and $\sigma e_{j}=\rho e_{i}$, hence $e_{i}$ and $e_{j}$ would form a 2 -loop (with the vertices $\rho e_{i}, \sigma e_{i}$ ), again contradicting the assumption on $F$.

Finally, to show that $G$ is alternating, note that, by (4.17) and (4.19), $G, F$ and $\varphi=\psi^{-1}$ satisfy the condition (4.1). Thus the incidence matrices (of vertices versus directed paths) associated with $G$ are among the incidence matrices (edges versus paths) associated with $F$, and hence unimodular. Especially then, the incidence matrix of the vertices versus all the directed paths of $G$ is unimodular, which, by the Hoffman-Kruskal Theorem (Theorem 4 in [4], cited in $\S 1$ of this note), implies that $G$ is necessarily alternating.

This completes proof of the theorem.

It is worth noting that the last part of the proof (namely that $G$ is alternating) can easily be established without using the result of [4] (which contains more than is needed here). 


\section{REFERENCES}

1. I. Heller and C. Tompkins, An extension of a theorem of Dantzig's, Ann. Math. Study No. 38.

2. I. Heller, On linear systems with integralvalued solutions, Pacific. J. Math., 7 (1957), 111-111.

3. - Constraint Matrices of Transportation-Type Problems, Naval Res. Logistics Quarterly, Vol. 4, No. 1.

4. A. J. Hoffman and J. B. Kruskal, Integral Boundary Points of Convex Polyhedra, Ann. Math. Study No. 38.

STANFORD UNIVERSITY

IBM RESEARCH CENTER 



\section{PACIFIC JOURNAL OF MATHEMATICS}

\section{EDITORS}

\author{
Ralph S. Phillips \\ Stanford University \\ Stanford, California \\ M. G. Arsove \\ University of Washington \\ Seattle 5 , Washington
}

\author{
A. L. Whiteman \\ University of Southern California \\ Los Angeles 7, California \\ Lowell J. Paige \\ University of California \\ Los Angeles 24, California
}
E. F. BECKENBACH
D. DERRY
ASSOCIATE EDITORS
T. M. CHERRY
M. OHTSUKA
H. L. ROYDEN
E. G. STRAUS
E. SPANIER
F. WOLF

\section{SUPPORTING INSTITUTIONS}

\author{
UNIVERSITY OF BRITISH COLUMBIA \\ CALIFORNIA INSTITUTE OF TECHNOLOGY \\ UNIVERSITY OF CALIFORNIA \\ MONTANA STATE UNIVERSITY \\ UNIVERSITY OF NEVADA \\ NEW MEXICO STATE UNIVERSITY \\ OREGON STATE UNIVERSITY \\ UNIVERSITY OF OREGON \\ OSAKA UNIVERSITY \\ UNIVERSITY OF SOUTHERN CALIFORNIA
}

\author{
STANFORD UNIVERSITY \\ UNIVERSITY OF TOKYO \\ UNIVERSITY OF UTAH \\ WASHINGTON STATE UNIVERSITY \\ UNIVERSITY OF WASHINGTON \\ AMERICAN MATHEMATICAL SOCIETY \\ CALIFORNIA RESEARCH CORPORATION \\ SPACE TECHNOLOGY LABORATORIES \\ NAVAL ORDNANCE TEST STATION
}

Mathematical papers intended for publication in the Pacific Journal of Mathematıcs should be typewritten (double spaced), and the author should keep a complete copy. Manuscripts may be sent to any one of the four editors. All other communications to the editors should be addressed to the managing editor, L. J. Paige at the University of California, Los Angeles 24, California.

50 reprints per author of each article are furnished free of charge; additional copies may be obtained at cost in multiples of 50 .

The Pacific Journal of Mathematics is published quarterly, in March, June, September, and December. Effective with Volume 13 the price per volume (4 numbers) is $\$ 18.00$; single issues, $\$ 5.00$. Special price for current issues to individual faculty members of supporting institutions and to individual members of the American Mathematical Society: $\$ 8.00$ per volume; single issues $\$ 2.50$. Back numbers are available.

Subscriptions, orders for back numbers, and changes of address should be sent to Pacific Journal of Mathematics, 103 Highland Boulevard, Berkeley 8, California.

Printed at Kokusai Bunken Insatsusha (International Academic Printing Co., Ltd.), No. 6, 2-chome, Fujimi-cho, Chiyoda-ku, Tokyo, Japan.

PUBLISHED BY PACIFIC JOURNAL OF MATHEMATICS, A NON-PROFIT CORPORATION

The Supporting Institutions listed above contribute to the cost of publication of this Journal, but they are not owners or publishers and have no responsibility for its content or policies. 


\section{Pacific Journal of Mathematics}

\section{Vol. 12 , No. 4 \\ April, 1962}

Tsuyoshi Andô, On fundamental properties of a Banach space with a cone ..... . 1163

Sterling K. Berberian, A note on hyponormal operators ................ 1171

Errett Albert Bishop, Analytic functions with values in a Frechet space . . . . . . . 1177

(Sherman) Elwood Bohn, Equicontinuity of solutions of a quasi-linear equation ............................................ 1193

Andrew Michael Bruckner and E. Ostrow, Some function classes related to the class of convex functions . . . . . . . . . . . . . . . . . . . . . . . . 1203

J. H. Curtiss, Limits and bounds for divided differences on a Jordan curve in the complex domain . . ................................. 1217

P. H. Doyle, III and John Gilbert Hocking, Dimensional invertibility . . . . . . . . 1235

David G. Feingold and Richard Steven Varga, Block diagonally dominant matrices and generalizations of the Gerschgorin circle theorem ................. 1241

Leonard Dubois Fountain and Lloyd Kenneth Jackson, A generalized solution of the boundary value problem for $y^{\prime \prime}=f\left(x, y, y^{\prime}\right) \ldots \ldots \ldots \ldots \ldots \ldots \ldots \ldots \ldots$

Robert William Gilmer, Jr., Rings in which semi-primary ideals are primary. . . . . 1273

Ruth Goodman, K-polar polynomials .......................... 1277

Israel Halperin and Maria Wonenburger, On the additivity of lattice completeness ........................................... 1289

Robert Winship Heath, Arc-wise connectedness in semi-metric spaces . . . . . . . . 1301

Isidore Heller and Alan Jerome Hoffman, On unimodular matrices . . . . . . . . . . . 1321

Robert G. Heyneman, Duality in general ergodic theory . . . . . . . . . . . . . . . 1329

Charles Ray Hobby, Abelian subgroups of p-groups . . . . . . . . . . . . . . . 1343

Kenneth Myron Hoffman and Hugo Rossi, The minimum boundary for an analytic

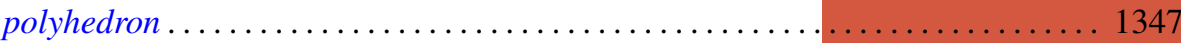

Adam Koranyi, The Bergman kernel function for tubes over convex cones ........ 1355

Pesi Rustom Masani and Jack Max Robertson, The time-domain analysis of a continuous parameter weakly stationary stochastic proces.

William Schumacher Massey, Non-existence of almost-complex structures on

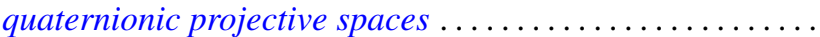

Deane Montgomery and Chung-Tao Yang, A theorem on the action of $\mathrm{SO}(3) \ldots . .1385$

Ronald John Nunke, A note on Abelian group extensions . . . . . . . . . . . . . 1401

Carl Mark Pearcy, A complete set of unitary invariants for operators generating finite $W^{*}$-algebras of type $I$

Edward C. Posner, Integral closure of rings of solutions of linear differential equations.

Duane Sather, Asymptotics. III. Stationary phase for two parameters with an application to Bessel functions.

J. Śladkowska, Bounds of analytic functions of two complex variables in domains

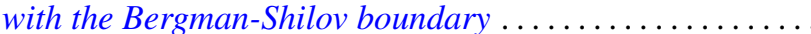

Joseph Gail Stampfli, Hyponormal operators .

George Gustave Weill, Some extremal properties of linear combinations of kernels

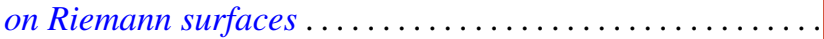

